\title{
Neonatal catecholamine levels and neurodevelopmental outcome: a cohort study
}

\author{
D J Evans, R J MacGregor, H G Dean, M I Levene
}

\begin{abstract}
Aims-To determine whether neonatal plasma catecholamine concentrations can be used to predict $(a)$ death plus disability and $(b)$ motor and cognitive impairment at 5 years of age.

Methods-A cohort comprised 136 preterm infants from two randomised controlled trials of neonatal sedation (19891992). Adrenaline (epinephrine) and noradrenaline (norepinephrine) were measured at baseline (first day) and 24 hours later. Intelligence and motor ability were assessed at 5-6 years.

Results-Infants who died or sustained disability had significantly higher plasma noradrenaline levels on the second day of life. Noradrenaline levels above $9.0 \mathrm{nmol} / 1$ were most predictive of death (likelihood ratio $3.27 ; 95 \%$ confidence interval 1.48 to 7.23) and death plus disability (likelihood ratio $3.55 ; 95 \%$ confidence interval 1.77 to 7.10). There was no correlation between neonatal catecholamine levels and cognitive or motor impairment at 5-6 years. Conclusions-Elevated noradrenaline levels are associated with adverse outcome in preterm infants; however, the power to predict death or disability is limited and they are not predictive of later motor or cognitive impairment.

(Arch Dis Child Fetal Neonatal Ed 2001;84:F49-F52)
\end{abstract}

Keywords: adrenaline; noradrenaline; catecholamines; intelligence; motor function; predictive ability

Preterm infants undergoing intensive care are capable of mounting a hormonal response to the stress of their illness and treatment, manifested by high catecholamine concentrations. ${ }^{1-4}$ In infants suffering from respiratory distress syndrome, adrenaline (epinephrine) and noradrenaline (norepinephrine) concentrations have been found to correlate with measures of illness severity. ${ }^{5}$ Extreme baseline catecholamine concentrations and a reduced noradrenaline response to opiate sedation are associated with subsequent mortality. ${ }^{5}$ There are no data on the usefulness of catecholamine levels for predicting disability in addition to mortality. It is also not known whether there is a correlation between neonatal catecholamine concentrations and cognitive or motor development in childhood.

Survivors of preterm birth are at risk of developing neurodevelopmental and, in particular, motor impairment. If catecholamines correlate with disease severity, they may be expected to correlate with scores of motor, and possibly cognitive, impairment. A study was therefore undertaken to examine the following questions. Are elevated neonatal catecholamine levels associated with death and disability, and, if so, what is their predictive ability? Do neonatal catecholamine levels correlate with measures of motor and cognitive impairment in childhood?

\section{Methods}

A cohort study was constructed from two randomised controlled trials of morphine sedation in ventilated preterm infants. The two trials were undertaken to determine the effects of opiate analgesia on the neonatal stress response in preterm infants requiring mechanical ventilation. The first trial in $1989-1990^{6}$ randomised 95 ventilated infants of $\leqslant 34$ weeks gestation to receive morphine $(50-100 \mu \mathrm{g} / \mathrm{kg} / \mathrm{h})$ alone $(n=29)$, pancuronium alone $(n=28)$, or both morphine and pancuronium $(n=38)$. The second trial in 1991-1992 ${ }^{7}$ randomised 41 ventilated infants of $\leqslant 34$ weeks gestation and was a double blind controlled trial of morphine (loading dose $200 \mu \mathrm{g} / \mathrm{kg} /$ over two hours followed by $25 \mu \mathrm{g} / \mathrm{kg} / \mathrm{h} ; \mathrm{n}=21$ ) or $5 \%$ dextrose placebo solution $(n=20)$, infused at the same rate.

Blood samples ( $1 \mathrm{ml}$ whole blood) were taken on entry (baseline) into both trials - that is, before any sedation and within 24 hours of age- and 24 hours later (baseline +24 hours). From these samples, the separated plasma was stored at $-70^{\circ} \mathrm{C}$ until a radioenzymic assay for adrenaline and noradrenaline ${ }^{89}$ could be performed. The coefficients of variation between and within assays were $12 \%$ and $7 \%$ respectively. Samples from the same infant were analysed within the same assay.

The United Leeds Teaching Hospitals Trust ethics committee had approved both trials before they began, and informed parental consent was obtained for each infant enrolled.

The survivors of the two trials were traced and the children assessed at 5-6 years of age. Consent to assess the children was initially obtained from both the general practitioner and hospital consultant and then from the parents. Cognitive assessment was performed using the full scale Wechsler preschool and primary scale of intelligence (WPPSI-R) ${ }^{10}$, and motor development was assessed using the Movement assessment battery for children (Movement ABC). ${ }^{11}$ In children with major disability, tests were undertaken to the level of the child's ability. All the children were seen at their homes except for five who were seen at school, and all were assessed by one experienced paediatrician (RJM) who was blinded to 
Table 1 Characteristics of infants studied with complete neonatal catecholamine and follow up data

\begin{tabular}{|c|c|c|c|c|c|c|}
\hline & \multicolumn{2}{|c|}{ Intact survival } & \multicolumn{2}{|l|}{ Death } & \multicolumn{2}{|l|}{ Disablity } \\
\hline & Median & $I Q R$ & Median & $I Q R$ & Median & $I Q R$ \\
\hline \multicolumn{7}{|c|}{ Infants with outcome data } \\
\hline Number & 72 & & 41 & & 15 & \\
\hline Gestation (weeks) & 29 & $27-31$ & 27 & $25-28$ & 28 & $27-30$ \\
\hline Birth weight $(\mathrm{g})$ & 1320 & $960-1740$ & 890 & $745-1070$ & 1260 & $930-1380$ \\
\hline \multicolumn{7}{|c|}{ Infants with outcome data plus catecholamine data } \\
\hline Number & 55 & & 23 & & 13 & \\
\hline Gestation (weeks) & 30 & $27-31.75$ & 27 & $26-28.5$ & 29 & $27-30$ \\
\hline Birth weight $(\mathrm{g})$ & 1340 & $1025-1700$ & 900 & $800-1040$ & 1260 & $940-1420$ \\
\hline
\end{tabular}

the allocated treatment and neonatal catecholamine concentrations. Disability was defined as cerebral palsy, intelligence quotient (IQ) $\leqslant 70$, blindness, or severe sensorineural hearing loss requiring hearing aids.

Analyses were undertaken to determine whether elevated neonatal plasma adrenaline and noradrenaline concentrations were associated with death, and also death plus disability combined. Where a statistically significant association existed, the concentrations of adrenaline and noradrenaline with the greatest predictive ability were determined by plotting relative (receiver) operating characteristic curves. Predictive ability was expressed as a likelihood ratio and $95 \%$ confidence intervals.

An analysis was also performed to determine whether neonatal plasma catecholamine levels were correlated with cognitive or motor impairment in surviving children. Nonparametric statistical methods (Mann-Whitney

Table 2 Neonatal catecholamine responses

\begin{tabular}{|c|c|c|c|c|c|c|}
\hline & \multicolumn{6}{|c|}{ Median catecholamine concentration (nmolll) } \\
\hline & $\begin{array}{l}\text { All survivors } \\
(n=68)\end{array}$ & $\begin{array}{l}\text { Death } \\
(n=23)\end{array}$ & $p$ Value & $\begin{array}{l}\text { Intact } \\
\text { survivors } \\
(n=55)\end{array}$ & $\begin{array}{l}\text { Death E } \\
\text { disability } \\
(n=36)\end{array}$ & $p$ Value \\
\hline \multicolumn{7}{|l|}{ Adrenaline } \\
\hline Baseline & 1.59 & 1.65 & 0.22 & 1.29 & 2.14 & 0.02 \\
\hline Baseline $+24 \mathrm{~h}$ & 0.99 & 1.34 & 0.08 & 0.96 & 1.30 & 0.06 \\
\hline Change over $24 \mathrm{~h}$ & -0.44 & -0.38 & 0.90 & -0.32 & -0.68 & 0.08 \\
\hline \multicolumn{7}{|l|}{ Noradrenaline } \\
\hline Baseline & 4.03 & 5.80 & 0.02 & 3.71 & 5.95 & 0.02 \\
\hline Baseline $+24 \mathrm{~h}$ & 3.12 & 9.27 & 0.0002 & 3.09 & 5.15 & 0.003 \\
\hline Change over $24 \mathrm{~h}$ & -0.67 & +1.06 & 0.01 & -0.49 & -0.14 & 0.21 \\
\hline
\end{tabular}

Comparisons are between those who died and the survivors, and between infants who survived without disability (intact) and those who died or were disabled. p Values were obtained with the Without disability (intact)
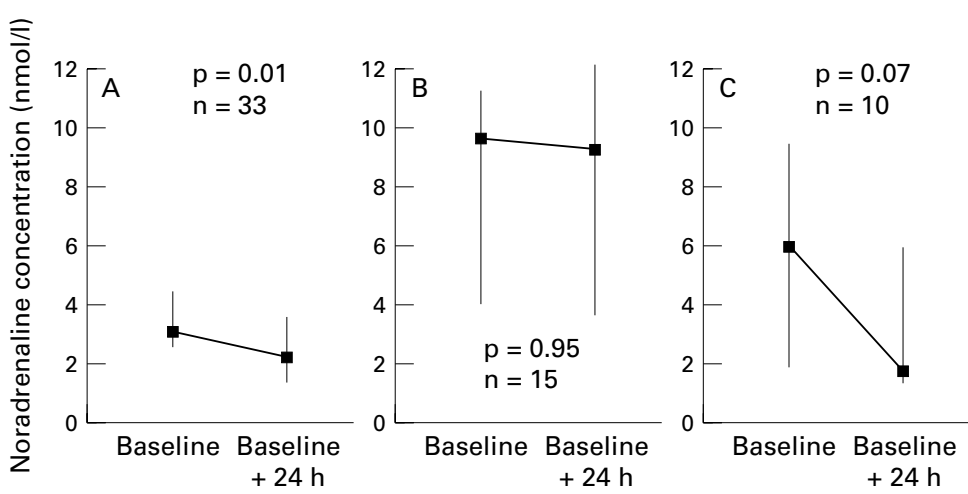

Figure 1 Noradrenaline response to morphine in the subgroup of 58 infants who received morphine sedation. (A) intact survivors; (B) infants who died; (C) infants who died plus those who were disabled. Morphine treatment was begun after the baseline sample and was continuing during the baseline +24 hour sample. Median values are plotted with the interquartile ranges indicated. $p$ values refer to the Wilcoxon matched pairs signed rank sum test.
U test, Wilcoxon signed rank sum, and Spearman's rank correlation) were used because the catecholamine concentrations, IQ, and Movement $\mathrm{ABC}$ scores were found to be not normally distributed.

The number of infants required to be enrolled and studied was determined by the respective power calculations of the previous studies. $^{6712}$

\section{Results}

A total of 136 infants were recruited into the two randomised controlled trials, 95 of whom survived to 5-6 years of age. The characteristics of these infants has been previously described. ${ }^{6712}$ Of the 95 surviving children, 87 (92\%) had WPPSI-R and Movement ABC assessments. Of the eight children who were not seen, three could not be traced, for two of them permission to be assessed was refused, for two no reply was received to three letters, they lived outside Leeds, and they were not on the phone, and one child came from a travelling family. Fifteen of the children assessed were disabled. Four of these were unable to undergo the WPPSI-R or Movement ABC assessments because of severe disability. One child with quadriplegic cerebral palsy was only able to perform the verbal component of the WPPSI-R. Of the 11 disabled children able to complete fully the assessments, eight had cerebral palsy (two quadriplegia, four diplegia, two hemiplegia), one had severe learning difficulties, one had septo-optic dysplasia with learning difficulties, and one had Dubowitz syndrome and learning difficulties. All the children seen were included in the analyses. The children who could not be scored on IQ and motor assessments were given a nominal score of 30 for the WPPSI-R and the maximum score of 40 for the Movement ABC.

Complete neonatal catecholamine data were available for $68(78 \%)$ of the surviving assessed children and $23(56 \%)$ of the infants who died. Table 1 gives the gestational age and birth weights of the 128 infants with outcome data (41 deaths and 87 follow up) and the 91 infants with outcome data plus complete catecholamine data.

Table 2 gives the median plasma adrenaline and noradrenaline concentrations at baseline and baseline +24 hours and the change in concentration over 24 hours. Generally, catecholamine concentrations were higher in infants who subsequently died or were found to have disability. The greatest statistical differences in concentrations were seen with noradrenaline at baseline +24 hours. Noradrenaline levels were highest in infants who died $(\mathrm{p}=0.0002)$ and remained high in the death plus disability group ( $p=0.003$; table 2$)$. Catecholamine concentrations decreased over the 24 hour neonatal study period except noradrenaline levels in those infants who died. In these infants, the noradrenaline levels actually rose, presumably reflecting a greater severity of disease. In the subgroup analysis of neonates receiving morphine, there was evidence that a reduced noradrenaline response to opiate 

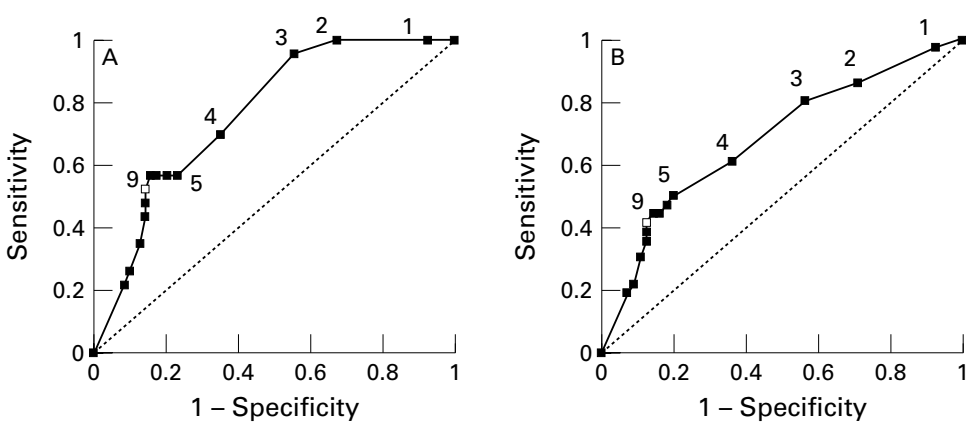

Figure 2 Relative (receiver) operating characteristic curves for different concentrations of noradrenaline at baseline +24 hours, in the prediction of $(A)$ death and $(B)$ death plus disability. Concentrations indicated next to data points are in units of nmol/l.
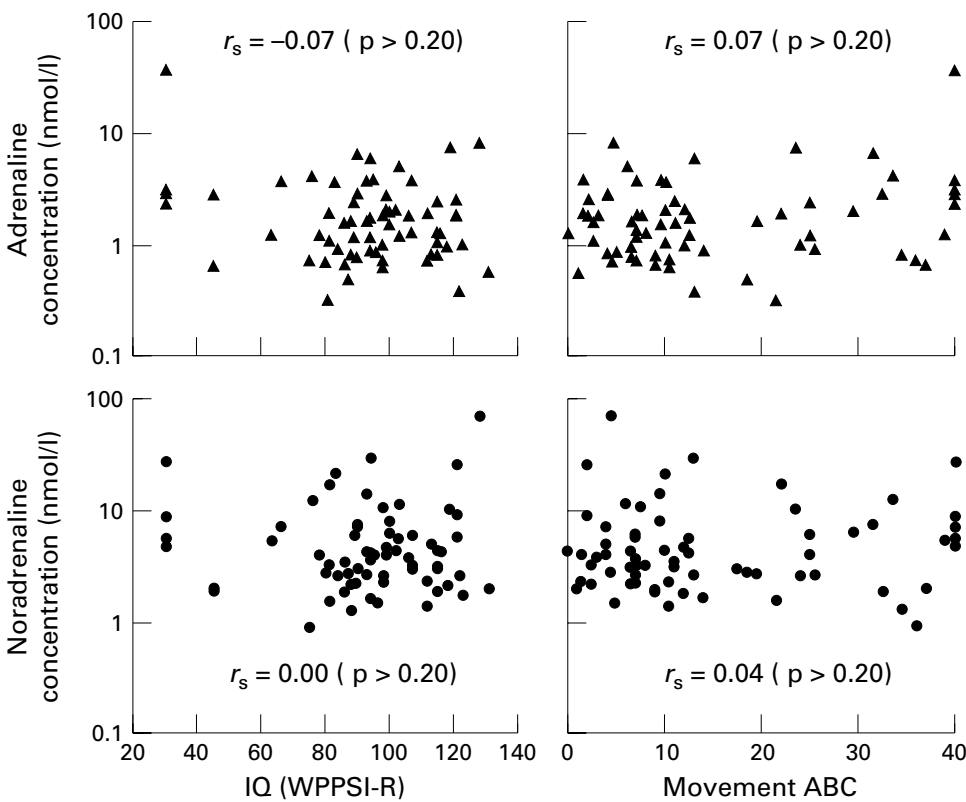

Figure 3 Scatter plots of baseline adrenaline and noradrenaline concentrations against IQ (Wechsler preschool and primary scale of intelligence (WPPSI-R)) scores and Movement assessment battery for children (Movement ABC) scores in the 68 surviving children. $r$, Spearman's rank correlation coefficient.

sedation existed in the infants who subsequently died (fig 1).

From the relative operating characteristic curves, the greatest predictive ability is associated with a noradrenaline at baseline +24 hours above $9.0 \mathrm{nmol} / 1$ (fig 2). The likelihood ratio for the prediction of death is $3.27(95 \%$ confidence interval (CI) 1.48 to 7.23 ), and for death plus disability it is 3.55 (95\% CI 1.77 to 7.10).

In the 68 surviving children, there was no evidence of an association between baseline catecholamines and scores of intelligence (WPPSI-R) or motor impairment (Movement ABC), as measured by Spearman's rank correlation (fig 3). Neither was there any association between the WPPSI-R or Movement ABC and catecholamine concentrations at baseline +24 hours or the change in catecholamine concentrations over the 24 hours.

\section{Discussion}

This study confirms previously published data suggesting an association between elevated neonatal catecholamine levels and mortality. It also suggests that both infants who die and those who develop disability have elevated neonatal catecholamine levels. This study goes further in critically examining the relation between high catecholamines and both death and disability from a viewpoint of clinical utility. A noradrenaline concentration above 9.0 $\mathrm{nmol} / \mathrm{l}$ is predictive of both death and death plus disability (fig 2). Despite the statistically significant likelihood ratios, however, the magnitudes of the ratios are low-that is, the odds after the test are only three times those before the test-and the lower limits of the $95 \%$ CI are close to unity. In comparison, the likelihood ratio of a major ultrasound abnormality in the prediction of death or disability in a similar preterm population is 3.9 (95\% CI 2.5 to $6.1) .{ }^{13}$ A larger study would allow more precision surrounding the estimate of the likelihood ratio. If neonatal catecholamines were to perform at least as well as ultrasound-that is, taking a ratio of 2.5 as the lowest acceptable likelihood ratio-a sample size of 770 infants would be required. Thus it is unlikely that catecholamine concentrations will ever be shown to be clinically useful in the prediction of death or disability.

Barker and Rutter ${ }^{5}$ suggest that a reduced noradrenaline response to opiate sedationthat is, a persistently high noradrenaline level despite six hours of sedation-was associated with increased mortality. This was confirmed in this study, although there was no evidence that a reduced noradrenaline response to sedation over 24 hours was associated with disability (fig 1).

The most severely ill infants are more likely to receive inotropes, the metabolites of which may raise blood noradrenaline concentrations. ${ }^{14}$ It is therefore possible that changes in adrenaline and noradrenaline are masked or altered by inotropic support, such as dopamine treatment. This appears not to be the case, however, because a review of the treatment charts shows that only two infants in the death and disability group and four in the intact survival group received dopamine treatment or adrenaline for resuscitation.

Survivors of preterm birth are at risk of developing neurodevelopmental and, in particular, motor impairment. If catecholamines correlate with disease severity, they may be expected to correlate with scores of motor, and possibly cognitive, impairment. Neither adrenaline nor noradrenaline concentrations were correlated with either Movement ABC scores or IQ. It is unlikely that either attrition or the assessments having poor discriminatory power are reasons for a potential correlation being missed by this study. Over $90 \%$ of the surviving children have been traced and assessed as part of this study, and one experienced neurodevelopmental paediatrician assessed all the children. Both assessments produced a wide range of scores. Some $20 \%$ of the surviving cohort assessed had significant disability, as previously defined. Although this is somewhat higher than many reports, this reflects the cohort's high risk nature because of the prerequisite for mechanical ventilation. 
Although there is some evidence that high catecholamine levels in the neonatal period are associated with death and disability, there is no evidence to suggest that they have any prognostic significance in terms of motor impairment or cognitive ability. It is still unclear whether high catecholamine concentrations are, in themselves, detrimental or whether they represent an adaptive response. There is a theoretical concern that measures to reduce catecholamines, such as opiate sedation, may blunt this adaptive response and lead to higher mortality and morbidity in surviving infants. From clinical studies, however, the reductions in catecholamine levels associated with morphine treatment ${ }^{12}$ and antenatal corticosteroids ${ }^{16}$ do not appear to be associated with a detrimental effect.

In summary, this study describes an association between elevated catecholamine levels and adverse outcome in preterm infants; however, the power to predict death or disability is limited. Neonatal catecholamine concentrations do not correlate with measures of motor or cognitive impairment in surviving children.

This work was supported by funding from Action Research. We are grateful to Mrs Jenny Wild for assistance with tracing the children.

1 Anand KJS, Sippell WG, Aynsley-Green A. Randomised trial of fentanyl anaesthesia in preterm babies undergoing surgery: effects on the stress response. Lancet 1987;i:243-8

2 Greisen G, Frederiksen PS, Hertel J, Christensen NJ. Catecholamine response to chest physiotherapy and endotracheal suctioning in preterm infants. Acta Paediatr Scand 1985;74:525-9.
3 Mehandru PL, Assel BG, Nuamah IF, Fanaroff AA, Kalhan SC. Catecholamine responses at birth in preterm newborns. Biol Neonate 1993;64:82-8.

4 Lagercrantz H, Bistoletti P. Catecholamine release in the newborn infant at birth. Pediatr Res 1977;11:889-93.

5 Barker DP, Rutter N. Stress, severity of illness, and outcome in ventilated preterm infants. Arch Dis Child Fetal Neonatal Ed 1996;75:F187-90.

6 Quinn MW, Otoo F, Rushforth JA. Effect of morphine and pancuronium on the stress response in ventilated preterm infants. Early Hum Dev 1992;30:241-8.

7 Quinn MW, Wild J, Dean HG, et al. Randomised double-blind controlled trial of effect of morphine on catecholamine concentrations in ventilated pre-term babies. Lancet 1993;342:324-7.

8 Morrison JKJ, Pearson SB, Dean HG. Parasympathetic nervous system in nocturnal asthma. BMf 1988;296:1427-9.

9 da Prada M, Zurcher G. Simultaneous radio enzymatic determination of plasma and tissue adrenaline, noradrenaline and dopamine within the femtomole range. Life Sci 1974:19:1161-74.

10 Wechsler D. Wechsler Preschool and Primary Scale of Intelligence: Revised (British Amendments). Oxford: The Psychological Corporation, 1990.

11 Henderson SE, Sugden DA. Movement Assessment Battery for Children. Oxford: The Psychological Corporation, 1992.

12 MacGregor R, Evans D, Sugden D, Gaussen T, Levene M. Outcome at 5-6 years of prematurely born children who received morphine as neonates. Arch Dis Child 1998;79:F40-3.

13 Graham M, Levene MI, Trounce JQ, Rutter N. Prediction of cerebral palsy in very low birthweight infants: prospective ultrasound study. Lancet 1987;ii:593-6.

14 Stopfkuchen H, Racke K, Schworer H, Queisser-Luft A, Vogel K. Effects of dopamine infusion on plasma catecholamines in preterm and term newborn infants. Eur f Pediatr 1991;150:503-6.

15 Anand KJ, Barton BA, McIntosh N, et al. Analgesia and sedation in preterm neonates who require ventilatory support: results from the NOPAIN trial (Neonatal Outcome and Prolonged Analgesia in Neonates). Arch Pediatr Adolesc Med 1999;153:331-8.

16 Kallio J, Karlsson R, Toppari J, Helminen T, Scheinin M, Kero P. Antenatal dexamethasone treatment decreases plasma catecholamine levels in preterm infants. Pediatr Res 1998;43:801-7 